pulmonary arterial pressure, $\mathrm{mPAP} \geq 25 \mathrm{mmHg}$ ) in the absence of significant interstitial lung disease (ILD), based on high resolution computed tomography and lung function tests. Borderline PAH was defined as mPAP of $20-24 \mathrm{mmHg}$ in in the absence of significant ILD. Patients with pulmonary hypertension (PH) due to other causes were excluded. Descriptive statistics and logistic regression analyses were performed and tested by the goodness-of-fit test with area under the curve (AUC).

Results: Mean age at onset was $54 \pm 14.1$, at $\mathrm{RHC} 61 \pm 10.9$ years. The time from sera to $\mathrm{RHC}$ was $0.9 \pm 3.1$ years. In total, $73.7 \%$ (123) patients had limited cutaneous SSc, $46.7 \%{ }^{48}$ were positive for anti-centromere antibody and $80.2 \%$ (134) were females. Of these, $123(73.7 \%)$ patients were included in the study, 28 patients with $\mathrm{PAH}, 45$ borderline $\mathrm{PAH}$ and 50 patients with no PH. Mean VEGF-A, C,D, CCL21 and Ang2 levels are shown in figure 1A. VEGF-A was significantly higher in SSc patients compared to healthy controls $(p=0.001)$, no significant differences between PAH and no PH patients were found. VEGF-D was decreased in SSc-PAH but not significantly. CCL21 and Ang2 levels were increased in patients with $\mathrm{PAH}$, whereas in VEGF-C was significantly decreased in patients with $\mathrm{PAH}$ (figure $1 \mathrm{~B}-\mathrm{D})$ ). In univariable logistic regression analyses, VEGF-C (OR $0.99,95 \% \mathrm{Cl} 0.997-0.998, \mathrm{p}=0.001, \mathrm{AUC}=0.79)$, CCL21 (OR 1, 95\% Cl 1-1.003, $\mathrm{p}=0.050, \mathrm{AUC}=0.69$ ) and Ang2 (OR 1, 95\% Cl 1-1.0001, $\mathrm{p}=0.49, \mathrm{AUC}=0.67$ ) were associated with $\mathrm{PAH}$ compared to no $\mathrm{PH}$ patients. Due to few event numbers, no multivariable analyses were performed.
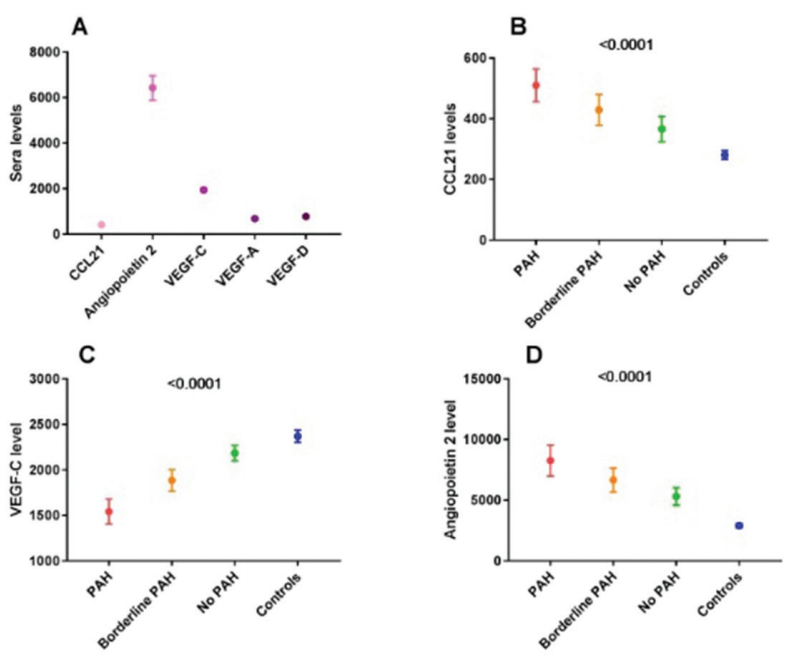

Abstract FRI0431 - Figure 1. Serum levels of A) all; B) CCL21; C) VEGF-C and D) Ang2

Conclusions: The present study is the first to demonstrate dysregulation of lymphangiogenetic factor expression of multiple targets in sera of SSc-PAH patients. Disclosure of Interest: None declared

DOI: 10.1136/annrheumdis-2018-eular.4972

\section{FRI0432 SINGLE-PORT THORACOSCOPIC SYMPATHICOTOMY FOR TREATMENT RESISTANT RAYNAUD'S PHENOMENON. FIRST REPORT OF A NOVEL MINIMALLY-INVASIVE ENDOSCOPIC TECHNIQUE}

A. Van Roon ${ }^{1}$, M. Kuijpers ${ }^{2}$, A. Eman Abdulle ${ }^{1}$, S. Van de Zande ${ }^{1}$, M. Mariani ${ }^{2}$, R. Bos ${ }^{3}$, T. Klinkenberg ${ }^{2}$, H. Bootsma ${ }^{4}$, A. Smit ${ }^{1}$, D.J. Mulder ${ }^{1} .{ }^{1}$ Dept. Internal Medicine, div. Vascular Medicine; ${ }^{2}$ Dept. Thoracic surgery, University of Groningen, University Medical Center Groningen, Groningen; ${ }^{3}$ Dept. Rheumatology, Medisch Centrum Leeuwarden, Leeuwarden; ${ }^{4}$ Dept. Rheumatology and Clinical Immunology, University of Groningen, University Medical Center Groningen, Groningen, Netherlands

Background: Raynaud's phenomenon of the hands is a great burden in daily life and reduces quality of live in patients with or without an underlying connective tissue disease. Although vasodilatory treatment may be effective in some patients, complaints may be resistant to treatment, for which additional treatment options are very limited. In earlier years, thoracic sympathectomy by anterior or axillary thoracotomy has been shown effective, but with a great surgical burden and limited durability. In the $90 \mathrm{~s}$, endoscopic procedures were introduced. However, these techniques needed multiple endoscopic ports and still performed a relatively mutilating sympathectomy of the sympathetic ganglia. In our centre, the singleport thoracoscopic sympathicotomy (SPTS) was developed, which is a new minimally invasive endoscopic technique with a limited surgical burden.

Objectives: The aim of the current pilot study was to evaluate feasibility and efficacy of SPTS in patients with treatment resistant Raynaud's.

Methods: This new technique entails only a single-port endoscopic procedure, during which a sympathicotomy (figure 1) of the nerve is performed, thus sparing the ganglia. Hospital stay is limited to one day. The procedure has been developed for treatment of hyperhidrosis and performed in our hospital in over 550 patients without major adverse events with a success rate of $98 \%$. In the current study, we aim to include 10 patients with treatment resistant Raynaud's, defined as unsatisfactory effect or contraindications of oral vasodilatory agents and iv prostaglandin analogous. Sympathicotomy was performed on the left hand first and the effects were compared with the contralateral hand after 3 and 12 months. Major exclusion criteria were severe lung involvement or proximal vascular stenosis. The primary end point was Raynaud's Condition Score (RCS) and Quality of life (SF-36). Among others, cooling fingertip plethysmography (PPG) and laser doppler imaging (LDI) were used as secondary end points for objective assessment of hand perfusion.

Results: This is an interim report, and 4 patients have been included so far (age $20,29,32,56$ years), male/female $3 / 1,3$ primary, 1 secondary), without a history of digital ulcers. No adverse events have been observed, apart from some hyperaemia in the first post-operative week. RCS significantly in each patient. Additionally, a clear improvement in hand perfusion following cooling was observed with PPG and LDI, as compared to the contralateral site. All 4 patients were satisfied and agreed to have their contralateral site operated on also. Patients will be followed in the outpatient clinic to assess long term efficacy.

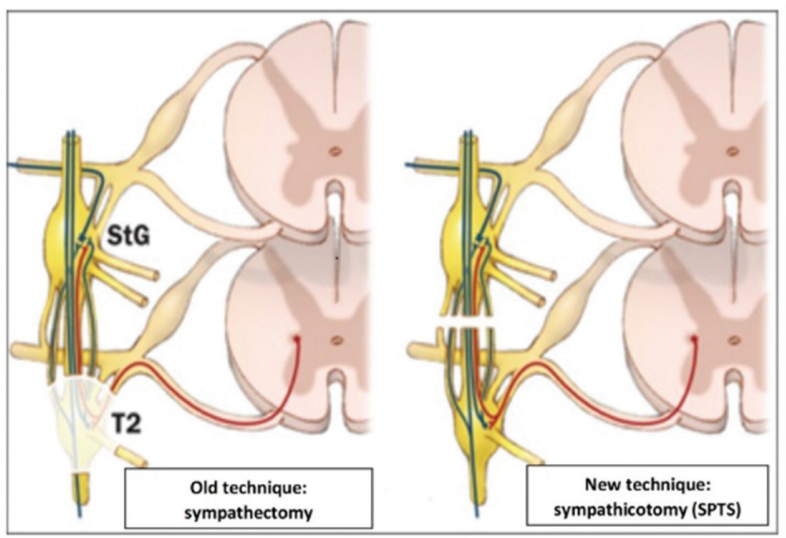

Conclusions: Single-port thoracoscopic sympathicotomy is a novel minimally invasive technique which appears to be safe and feasible in patients with treatment resistant Raynaud's phenomenon. However, this study is ongoing and longterm efficacy needs to be established.

Disclosure of Interest: None declared

DOI: 10.1136/annrheumdis-2018-eular.6991

\section{FRI0433 ASSESSING MORTALITY MODELS IN SYSTEMIC SCLEROSIS RELATED INTERSTITIAL LUNG DISEASE}

R.L. Mango ${ }^{1}$, E.L. Matteson ${ }^{1}$, C.S. Crowson ${ }^{1}$, J.H. Ryu ${ }^{2}$, A. Makol ${ }^{1} .{ }^{1}$ Division of Rheumatology, ${ }^{2}$ Division of Pulmonary and Critical Care, Mayo Clinic, Rochester, USA

Background: Interstitial lung disease (ILD) is a major cause of morbidity and mortality in systemic sclerosis (SSc). However, the severity of lung involvement can vary widely, and current evidence-based treatment options have modest benefit. Prognosis assessment is particularly important for initial management decisions at the time SSc-ILD is diagnosed. There are now multiple mortality models available for use in SSc-ILD which utilise patient's baseline parameters

Objectives: The Gender, Age, and Lung Physiology (GAP) model, ${ }^{1}$ interstitial lung diseases -GAP (ILD-GAP) model, ${ }^{2}$ and the Smoking history, Age, and Diffusion capacity of the Lung (SADL) model ${ }^{3}$ were compared using a systemic sclerosis-ILD (SSc-ILD) cohort to evaluate which best determined prognosis.

Methods: The models were applied to a cohort of patients with SSc (meeting the 2013 ACR/EULAR classification criteria) seen at a tertiary care centre within 1 year of ILD diagnosis from 2000-2013. Demographics, clinical characteristics, 
and mortality were recorded. The performance of the models was assessed using standardised mortality ratios (SMR) of observed vs. predicted outcomes for calibration, and concordance (c)-statistics for discrimination.

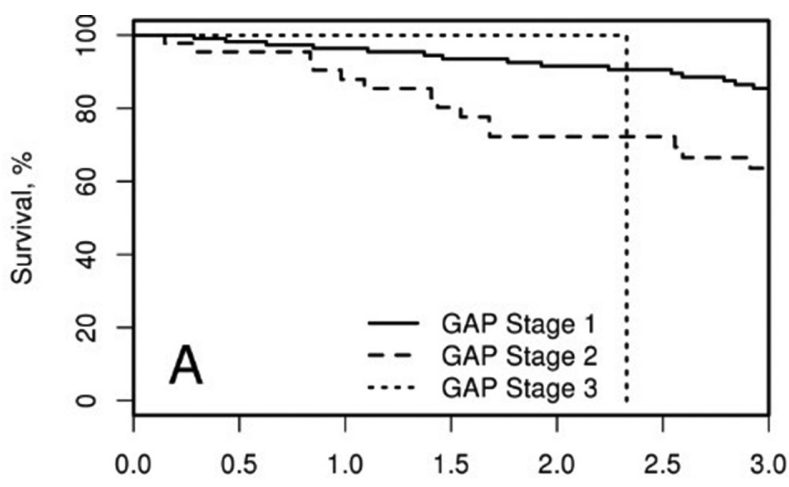

Years since ILD diagnosis

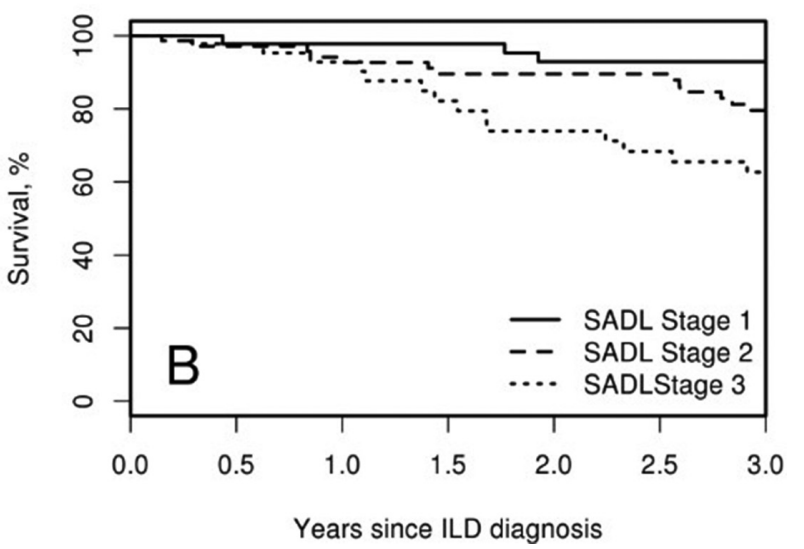

Results: The cohort included 179 patients with SSc-ILD. Mean age at ILD diagnosis was 57.8 years. There was a female predominance $(73 \%)$. The mean length of follow up after ILD diagnosis was 4.3 years (SD 4.0). Based on high resolution chest CT (or biopsy when available), $147(83 \%)$ were characterised as nonspecific interstitial pneumonia (NSIP), 31 (17\%) as usual interstitial pneumonia (UIP), 1 as unclassifiable ILD. A history of smoking was noted in $49 \%$, and this was not associated with ILD pattern (former/current $46 \%$ in NSIP vs. $61 \%$ in UIP, $p=0.11$ ). Pulmonary hypertension (PHTN) was noted in $66(49 \%)$ at baseline, and this did not differ between ILD subtypes. $84 \%$ had limited cutaneous SSc, $9 \%$ had diffuse cutaneous, and 7\% SSc sine scleroderma. SSc specific serologies (i.e., SCL-70, centromere, and/or RNA Pol III) were positive in 73 (43\%) patients, somewhat more common in NSIP than UIP ( $47 \%$ vs $26 \%$; $p=0.034)$. During a median of 3.2 years of follow-up, 65 patients died. SSc-ILD patients with UIP had a higher mortality than those with NSIP (hazard ratio: 2.27 ; 95\% Cl: 1.03-4.97). Other risk factors for progression included baseline DLCO $(p<0.001)$, FVC $(p<0.001)$ and PHTN $(p=0.012)$. All 3 models had comparable discrimination $(c=0.72,0.72$, and 0.70 , respectively). Figure 1 shows the differential mortality based on the GAP and SADL staging systems. (Note the staging in the GAP and ILD-GAP models are identical.) Regarding calibration, the ILD-GAP model underestimated mortality (SMR: 1.5; 95\% Cl: 1.05-2.14). Calibration was acceptable for SADL (SMR: 0.77; 95\% Cl: 0.54-1.10) and GAP (SMR: 0.90; 95\% Cl: 0.63-1.29). The SADL model overestimated mortality in Stage III ILD.

Conclusions: The ILD-GAP model underestimated mortality, and the SADL model overestimated mortality in certain subgroups. However, the GAP model performed well in this cohort, providing the best prognostic information for SScILD.
REFERENCES:

[1] Ley B, et al. Annals of internal medicine 2012;156(10):684-691.

[2] Ryerson CJ, et al. Chest 2014;145(4):723-728.

[3] Morisset J, et al. Chest 2017;152(5):999-1007.

Disclosure of Interest: None declared

DOI: 10.1136/annrheumdis-2018-eular.3331

\section{FRI0434 PATIENTS WITH INFLAMMATORY MYOPATHIES ADMITTED IN ICU ARE CHARACTERISED BY RECENT ONSET AND UNTREATED ACTIVE DISEASE AS WELL AS OLDER AGE AND HIGH COMORBIDITIES}

B. Michard, T. Artzner, F. Severac, M. Schenck, J.-E. Herbrecht, R. JanssenLangenstein, O. Collange, G. Freys, J. Pottecher, J. Sibilia, T. Martin, F. Meziani, F. Schneider, A. Meyer. CHU Strasbourg, Strasbourg, France

Background: Inflammatory myopathies (IM) are life-threatening but treatable diseases. The risk factors for admission in Intensive care unit (ICU), the management and the outcome of patients with IM admitted to ICU has not yet been assessed.

Objectives: To assess the clinical features, risk factors and outcome of patients with IM admitted in ICU.

Methods: A single centre cohort of 509 patients with IM was screened for admission in ICU from 1992 to 2017. Patients admitted for trauma or for complications from elective surgery were excluded. Control patients with IM who had not been hospitalised in ICU were randomly selected from the cohort.

Results: Thirty-two ICU admissions were recorded in $27 \mathrm{IM}$ patients during the study period $(<0.05 \%$ of admissions over this 25 year period). Three IM patients were admitted more than once.

Characteristics and prognosis of patients in ICU

Patients hospitalised in ICU had a mean age of $63 y+ \pm 15$ with SAPS II score of 58 \pm 24 and LODS score of $9 \pm 5$ corresponding to an intermediate severity at admission in ICU. The delay between IM diagnosis and first ICU admission was $27 \pm 43$ months. It is noteworthy that 12 patients $(44 \%)$ were admitted in the ICU within the first month of IM diagnosis, among whom 4 (15\%) were diagnosed with IM during ICU stay. Sixteen patients $(60 \%)$ were not treated at the time of their first ICU admission. In $56 \%$ of the ICU stays, patients had active disease at admission. Patients were most frequently admitted for respiratory failure $(88 \%)$ but cardiac $(47 \%)$, renal $(47 \%)$, neurologic $(47 \%)$, haematological $(22 \%)$ and hepatic $(15 \%)$ failures were also recorded. Infections were present in $72 \%$ of the ICU stays, with septic shock in $44 \%$. Nine patients (33\%) died in ICU and 3 others (11\%) within 90 days of the last ICU discharge (vs. $15 \%$ during a $7.5 \pm 5$ years period of follow-up in the control group, $p<0.0001$ ).

Risk factors of ICU admission: The case control analysis identified 6 risk factors significantly associated with hospitalisation in the ICU, 3 of which were independently associated with hospitalisation in multivariate analysis. ICU patients had a higher age at first clinical signs of illness $(62 \pm 13$ years old vs. $53 \pm 13$ years old, $p<0.05)$, they had a higher rate of chronic kidney failure ( $26 \%$ vs. $0 \%, p<0.05)$ and higher incidence of arterial or venous thrombosis history $(37 \%$ vs. $0 \%$, $\mathrm{p}<0.05)$. Other risk factors that were only identified in univariate analysis included lower BMI (22.6 \pm 4.5 vs. $25.4 \pm 6.3)$, a history of interstitial lung disease ( $48 \%$ vs. $30 \%$ ) and a higher Charlson comorbidity index (4.6 \pm 2.6 vs. $3.3 \pm 2$ ).

The type of myositis was not significantly associated with admission in ICU, although no patient admitted in ICU had sIBM. It is noteworthy that cumulative number of immunomodulatory treatments in patients at the time of hospitalisation in ICU was lower than in the control group ( $0.5 \pm 0.7$ vs. $1.9 \pm 0.8 p<0.001)$.

Conclusions: IM patients admitted in ICU frequently have recent onset and untreated active IM with respiratory failure. Admission to ICU is associated with older age and a higher number of comorbidities. Mortality of IM patients in ICU is high.

Disclosure of Interest: None declared

DOI: 10.1136/annrheumdis-2018-eular.6148 